\title{
Recovery of Decreased Metabotropic Glutamate Receptor 5 Availability in Abstinent Alcohol-Dependent Patients
}

\author{
Jenny Ceccarini ${ }^{* 1,2}$, Gil Leurquin-Sterk ${ }^{* 1,2}$, Cleo Lina Crunelle ${ }^{3,4}$, Bart de Laat ${ }^{1,2,5}$, Guy Bormans ${ }^{6}$, Hendrik Peuskens ${ }^{7,8}$, \\ and Koen Van Laere ${ }^{1,2,5}$ \\ ${ }^{1}$ Department of Nuclear Medicine and Molecular Imaging, UZ Leuven, Leuven, Belgium; ${ }^{2}$ Department of Imaging and Pathology, KU \\ Leuven, Leuven, Belgium; ${ }^{3}$ Toxicological Center, University of Antwerp, Wilrijk, Belgium; ${ }^{4}$ Department of Psychiatry, Universitair \\ Ziekenhuis Brussel, Brussels, Belgium; ${ }^{5}$ MoSAIC, Molecular Small Animal Imaging Center, KU Leuven, Leuven, Belgium; \\ ${ }^{6}$ Laboratory for Radiopharmacy, KU Leuven, Leuven, Belgium; ${ }^{7}$ University Psychiatric Center, KU Leuven, Kortenberg, Belgium; \\ and ${ }^{8}$ Kliniek Broeders Alexianen, Tienen, Belgium
}

Animal models of alcohol dependence and relapse demonstrate an important role of the glutamatergic system, in particular, cerebral metabotropic glutamate receptor 5 (mGluR5). ${ }^{18}$ F-3-fluoro-5-[(pyridin3-yl)ethynyl]benzonitrile ( $\left.{ }^{18} \mathrm{~F}-\mathrm{FPEB}\right)$ PET has revealed that chronic alcohol use leads to decreased limbic mGluR5 availability, which was associated with less craving. Here, we tested whether the state of decreased mGluR5 availability in alcohol-dependent patients normalizes during abstinence (at 2 and 6 mo of detoxification) and whether initial mGluR5 imaging parameters can predict individual relapse. Methods: ${ }^{18} \mathrm{~F}$-FPEB scans were performed for 16 recently detoxified alcohol-dependent patients (baseline condition), $2 \mathrm{mo}$ after detoxification $(n=10)$, and 6 mo after detoxification $(n=8)$; 32 age- and sex-matched controls were included for comparison. mGluR5 availability was quantified by the ${ }^{18} \mathrm{~F}-\mathrm{FPEB}$ total distribution volume using both voxel-by-voxel and volume-of-interest analyses. During follow-up, craving was assessed using the Desire for Alcohol Questionnaire, and alcohol consumption was assessed using the timeline follow-back method and monitored by hair ethyl glucuronide analysis. Results: During abstinence, alcohol-dependent patients showed sustained recovered mGluR5 availability in cortical and subcortical regions compared with the baseline, up to the levels observed in controls, after 6 mo in most areas except for the hippocampus, nucleus accumbens, and thalamus. Higher striatopallidal mGluR5 availability was observed at the baseline in patients who had a relapse during the $6-$ mo follow-up period (+25.1\%). Also, normalization of striatal mGluR5 to control levels was associated with reduced craving ("desire and intention to drink" and "negative reinforcement"; $r=0.72-0.94)$. Conclusion: Reduced cerebral mGluR5 availability in alcohol-dependent patients recovers during abstinence and is associated with reduced craving. Higher striatal mGluR5 availability in alcohol-dependent users may be associated with long-term relapse.

Key Words: mGluR5; alcohol addiction; alcohol abstinence; alcohol craving

J Nucl Med 2020; 61:256-262

DOI: 10.2967/jnumed.119.228825

Received Mar. 19, 2019; revision accepted Jul. 19, 2019.

For correspondence or reprints contact: Jenny Ceccarini, Department of Imaging and Pathology, KU Leuven, Herestraat 49, 3000 Leuven, Belgium. E-mail: jenny.ceccarini@uzleuven.be

${ }^{*}$ Contributed equally to this work.

Published online Sep. 3, 2019.

COPYRIGHT (C 2020 by the Society of Nuclear Medicine and Molecular Imaging.
$\mathbf{R}$ high rates of relapse and only a moderate effect of available therapies (1). Understanding the neurobiologic mechanisms that mediate successful recovery and identifying suitable biomarkers that may predict relapse or guide therapeutic interventions have become central in alcohol addiction outcome research. PET neuroimaging, in particular, holds promise because of its capacity to link molecular mechanisms and behavior (2). The highly selective and potent metabotropic glutamate receptor 5 (mGluR5) PET radioligand ${ }^{18} \mathrm{~F}$-3-fluoro-5-[(pyridin-3-yl)ethynyl]benzonitrile ( $\left.{ }^{18} \mathrm{~F}-\mathrm{FPEB}\right)(3-6)$ recently revealed that alcohol-dependent patients showed decreased cerebral mGluR5 availability in various cortical and subcortical limbic areas during the first $2 \mathrm{wk}$ of abstinence (7); this decrease was associated with higher alcohol consumption in the period preceding abstinence. Furthermore, decreasing mGluR5 function by specific pharmacotherapy, such as negative allosteric modulation or antagonism, might improve outcomes in abstinent alcoholdependent patients. Indeed, mGluR5 antagonists were able to prevent relapse in animal models $(8-10)$.

Additionally, although the anticraving effect of acamprosate was initially believed to be due in part to the inhibition of the $\mathrm{N}$ methyl-d-aspartate receptor and mGluR5 function (11), actions on these targets are not fully clear. On the other hand, the development of positive allosteric mGluR5 modulators has revealed that allosteric activation of this receptor might also have therapeutic benefits for the treatment of numerous central nervous system disorders, including drug addiction and deficits in extinction learning (12-15).

The reduced mGluR5 availability observed in alcohol-dependent patients could also represent a preexisting condition. Genetic studies have shown that individuals with specific mGluR5 polymorphisms are at higher risk for developing alcohol dependence $(16,17)$. Also, our previous PET work with healthy social drinkers revealed that higher mGluR5 availability was associated with more novelty seeking (18). Moreover, lower mGluR5 binding in alcohol-dependent patients was associated with less craving (7) and therefore with a lower relapse risk.

So far, to our knowledge, no longitudinal PET investigations of mGluR5 in alcohol-dependent patients or other substance use dependence have been reported. In a longitudinal rat model of alcohol consumption, ${ }^{18} \mathrm{~F}$-FPEB PET revealed that self-administration of alcohol resulted in decreased mGluR5 availability in the hippocampus and amygdala compared with the baseline (19). Similarly, mGluR5 
availability decreased during exposure to cocaine in the hippocampus (20). Regarding glutamate changes during withdrawal, to date, few studies have provided evidence for a hyperglutamatergic state during early abstinence that normalizes within $14 \mathrm{~d}(21,22)$. Additionally, a temporary increase in the glutamate concentration has been demonstrated after $8-12 \mathrm{~h}$ on acute forced alcohol administration $(21,23,24)$. Recent preclinical mGluR5 imaging data complemented these previous results, showing a decreased prefrontal glutamate concentration during alcohol or cocaine exposure that normalized after a few weeks of abstinence $(19,20)$; however, over longer abstinence periods, other findings showed a trend toward an increase in central glutamate levels $(25,26)$ or no changes $(27)$.

Here, we assessed cerebral mGluR5 availability in alcoholdependent patients at 2 and 6 mo after the cessation of alcohol consumption and compared it with mGluR5 availability during the recent detoxification (within $2 \mathrm{wk}$ of abstinence). Additionally, we investigated the relationship of imaging parameters to craving as well as the potential predictive value of baseline imaging parameters by examining whether regional initial mGluR5 availability in alcoholdependent patients would be different between patients who had a relapse during the 6-mo follow-up period and those who did not.

\section{MATERIALS AND METHODS}

\section{Participants}

The local university ethics committee approved this study, and all subjects signed a written informed consent form. The group of 16 alcohol-dependent patients included in the present study was the same as that in the study of Leurquin-Sterk et al. (7). In this subsequent study, all alcohol-dependent patients engaged in a standard detoxification program consisting of a 2 -wk inpatient period with medically supervised abstinence and then a regular follow-up by a board-certified psychiatrist specializing in addiction. In brief, exclusion criteria were substance use disorders other than alcohol, with the exception of nicotine dependence; any other psychiatric diseases; chronic use of benzodiazepines; and abnormal findings on physical examination, blood tests, urine toxicology, or structural MRI (7). The first ${ }^{18}$ F-FPEB PET scan (baseline condition) was performed within the first 2 weeks of medically supervised abstinence (7). Participants agreed to return at 2 and 6 mo after the start of the alcohol detoxification program for a follow-up visit including a physical examination, blood tests, urine toxicology, standard questionnaires, and structural MRI and ${ }^{18} \mathrm{~F}-\mathrm{FPEB}$ PET scans. Craving at these evaluation moments was assessed using the shortened, 13-item version of the Desire for Alcohol Questionnaire (28).

The sample of healthy controls $(n=32)$ represented a subsample from a previous study (18) and was randomly selected on the basis of age by a person who was unaware of both study protocols to obtain 2 age-matched controls for each patient. All controls were nonsmokers, had negative urine toxicology and normal structural MRI findings, were free of current or past psychiatric disorders (including substance use disorders), and reported low alcohol consumption ( $\leq 7$ units/wk); anyone reporting regular binge drinking ( $\geq 5$ units [unit is, for example, a glass of wine or beer] on 1 occasion) was excluded $(7,18)$.

\section{Assessment of Alcohol Consumption and Relapse During Follow-Up}

For the entire follow-up period, self-reported alcohol consumption was recorded daily using the timeline follow-back method (29). Relapse was defined when patients reported at least 1 drinking day with an alcohol consumption equivalent to that in the period before the start of the initial detoxification program and before the baseline scan. Effective reduction in alcohol consumption compared with the baseline condition was also objectively verified at the 2- and 6-mo follow-up visits by quantitative measurements of the alcohol metabolite hair ethyl glucuronide (30). As described previously (7), a 3-cm strand of scalp hair cut as proximally as possible was analyzed to obtain a quantitative measurement of alcohol consumption over the prior 3 mo.

\section{MRI and mGluR5 PET Imaging}

Brain MRI and PET scans were repeated at the 2- and 6-mo followup visits using the same procedures as for the baseline scans (Table 1) [ID]TBL1[/ID] $(3,18)$. Individual MR images were used for automatic volume-of-interest (VOI) determination and coregistration with PET images as well as for voxel-based morphometry (VBM) analysis. ${ }^{18} \mathrm{~F}-$ FPEB PET data were acquired on a HiRez Biograph 16-slice PET/CT camera (Siemens Inc.) between noon and 2 PM. A dynamic PET acquisition was performed for $90 \mathrm{~min}$ after a bolus injection of ${ }^{18} \mathrm{~F}$-FPEB (3).

PET data were analyzed using PMOD software (v3.605; PMOD Technologies) and statistical parametric mapping (SPM12). Parametric maps of ${ }^{18} \mathrm{~F}$-FPEB total distribution volume $\left(\mathrm{V}_{\mathrm{T}}\right)$ were generated using the Logan graphical approach (31) and were used as surrogates for mGluR5 availability. Additionally, a VOI analysis was performed using the N30R83 probabilistic atlas of Hammers et al. (32), in which $V_{T}$ values were derived from a reversible 2-tissue compartment model $(3,4)$. Because brain atrophy in alcohol-dependent patients might already be partially reversible during the first month of abstinence $(33,34)$, voxel- and VOIbased PET data were also calculated with partial-volume correction using the method of Müller-Gärtner et al. (35) and the geometric transfer matrix method (36), respectively. Effective changes in gray matter volumes at 2 and 6 mo of abstinence compared with the baseline condition were assessed using VBM analysis (with SPM12 and the VBM8 toolbox (7)).

\section{Statistical Analyses}

Statistical analyses were performed using Statistica version 12 (Statsoft). Normally distributed variables are reported as mean $\pm \mathrm{SD}$, and skewed variables are reported as median and interquartile range. Potential within-subject changes in mGluR5 availability at the 2- and 6-mo followup visits compared with the baseline condition were assessed at the voxel level using SPM12 with paired $t$ tests. For the SPM12 analysis, $\mathrm{V}_{\mathrm{T}}$ images were spatially smoothed using a gaussian kernel of $12 \mathrm{~mm}$. The SPM12 threshold was set at $p_{\text {height }}$ (voxel level) of $<0.001$, with a minimal cluster size extent of 200 voxels. Only significant clusters with $p_{\text {cluster }}$ (cluster level) of $<0.05$ (corrected for multiple comparisons) were retained.

VOI-based ${ }^{18} \mathrm{~F}$-FPEB $\mathrm{V}_{\mathrm{T}}$ values were used to investigate whether mGluR5 availability in alcohol-dependent patients during follow-up remained significantly lower than that in controls $(n=32$ (7)); 2-sided $t$ tests were used for independent samples. On the basis of previous evidence (37), regarding the group differences in mGluR5 availability between alcohol-dependent patients at baseline and controls, the results were controlled for smoking status (7) so that group differences in smoking did not affect the present mGluR5 findings. The results were controlled for smoking status using a multivariate general linear model with $\mathrm{V}_{\mathrm{T}}$ values as dependent variables and with group status and smoking status as independent predictors. No significant $\mathrm{V}_{\mathrm{T}}$ differences were observed between alcohol-dependent smokers and alcohol-dependent nonsmokers in any region. For the present study, the smoking patients did not change their amount of smoking during follow-up.

Moreover, independent-sample $t$ tests were used for comparing baseline $V_{T}$ values between patients with relapse and those without relapse, and Pearson correlations were used to estimate the association between baseline $\mathrm{V}_{\mathrm{T}}$ values and alcohol consumption during follow-up. Correlation analyses were performed between mGluR5 availability and changes in craving dimensions in regions showing a difference in baseline $V_{T}$ between patients with relapse and those without relapse. In addition, the effect of baseline $V_{T}$ values on alcohol consumption during follow-up was tested using a Poisson model for the outcome "number of drinks per week" and a linear model for the outcome "number of drinks per drinking day." 


\begin{tabular}{|c|c|c|c|c|}
\hline \multirow[b]{2}{*}{ Characteristic } & \multirow[b]{2}{*}{ Controls } & \multirow[b]{2}{*}{ Alcohol-dependent patients at baseline } & \multicolumn{2}{|c|}{$\begin{array}{l}\text { Patients abstaining from } \\
\text { alcohol at: }\end{array}$} \\
\hline & & & 2-mo follow-up & 6-mo follow-up \\
\hline No. of patients & 32 & 16 & 10 & 8 \\
\hline Age $(y)$ & $45 \pm 13$ & $45 \pm 8$ & $46 \pm 8$ & $44 \pm 8$ \\
\hline No. of women/men & $14 / 18$ & $3 / 13$ & $3 / 7$ & $2 / 6$ \\
\hline \multicolumn{5}{|l|}{ Alcohol craving (DAQ scores) } \\
\hline Desire and intention & & $16 \pm 7$ & $9 \pm 4$ & $10 \pm 4$ \\
\hline Negative reinforcement & & $14 \pm 7$ & $6 \pm 3$ & $9 \pm 7$ \\
\hline Control & & $5 \pm 3$ & $4 \pm 3$ & $7 \pm 5$ \\
\hline \multicolumn{5}{|l|}{ Alcohol consumption parameters } \\
\hline Reported alcohol use (y) & $26.6 \pm 8.1$ & $26.5 \pm 12.9$ & & \\
\hline Abstinence (d) & $5.8 \pm 6.6^{\star}$ & $7 \pm 4^{\dagger}$ & $56 \pm 12^{\star, \ddagger}$ & $101 \pm 20^{\star, \S}$ \\
\hline No. of drinks/wk (TLFB) & $2.4 \pm 2.1$ & $133(124-184)^{\|}$ & $1(0-4.1)^{\ddagger, \|}$ & $6(1-8)^{\S, \|}$ \\
\hline No. of drinks/drinking day (TLFB) & & $19(18-26)^{\|}$ & $2(0-10)^{\ddagger, \|}$ & $5(3-10)^{\S, \|}$ \\
\hline hEtG (pg/mg) & $8.1(5.3-17.5)^{\|, \text {, }}$ & $221(143-486)^{\|,, \#}$ & $51(9-118)^{\ddagger, \|}$ & $61(30-76)^{\S, \|}$ \\
\hline \multicolumn{5}{|l|}{${ }^{18} \mathrm{~F}-\mathrm{FPEB}$ imaging } \\
\hline Injected dose (MBq) & $177 \pm 7$ & $175 \pm 11$ & $174 \pm 7$ & $174 \pm 5$ \\
\hline Specific activity (MBq/nmol) & $90 \pm 52$ & $97 \pm 51$ & $81 \pm 38$ & $140 \pm 67$ \\
\hline Injected mass $(\mu \mathrm{g})$ & $0.63 \pm 0.60$ & $0.56 \pm 0.43$ & $0.58 \pm 0.24$ & $0.34 \pm 0.18$ \\
\hline \multicolumn{5}{|l|}{ 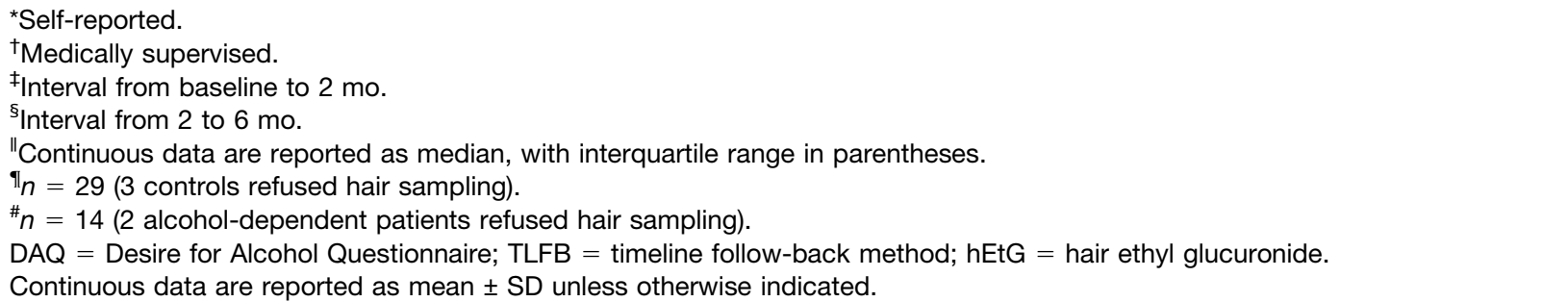 } \\
\hline
\end{tabular}

\section{RESULTS}

\section{Subjects and Follow-Up Data}

Of the 16 alcohol-dependent patients who were initially recruited for the baseline condition, 10 and 8 patients underwent the 2- and 6-mo follow-up evaluations, respectively (Table 1). Subjects who did not return for the study had a relapse documented by either a board-certified psychiatrist specializing in addiction or a family member during follow-up. Moreover, 2 patients had a relapse during the interval from 2 to 6 mo (i.e., resumed alcohol consumption corresponding to the 1 prior detoxification [interval from baseline to 2 mo]) and were consequently considered relapsers. Eleven alcohol-dependent patients were cigarette smokers (as reported in (7); average daily number of cigarettes, 18.5; Fagerström Test for Nicotine Dependence score, $5.5 \pm 2.7$ ). Among this group of smokers, 6 patients scanned at the 2- and 6-mo visits did not change their smoking pattern during follow-up (16.2 \pm 4.8 cigarettes per day).

\section{Changes in Brain Volumes and in mGluR5 Availability Over Time}

VBM and VOI-based analyses did not show changes in gray matter volumes at 2 and 6 mo of abstinence in comparison to the baseline condition (Supplemental Fig. 1). In a comparison of
${ }^{18} \mathrm{~F}-\mathrm{FPEB} \quad \mathrm{V}_{\mathrm{T}}$ calculated with partial-volume correction and obtained after 2 mo of abstinence to the baseline condition, the SPM12 analysis revealed significantly increased mGluR5 availability in large bilateral cortical and subcortical clusters, covering the hippocampus, parahippocampal gyrus, middle frontal gyrus, superior orbitofrontal gyrus, anterior cingulate cortex, insula, putamen, and left inferior temporal lobe ( $p_{\text {height }}<0.001 ; t>5.7$ ) (Fig. 1A). When the statistical T-maps were interrogated at the most stringent peak voxel level of $p_{\text {height(family-wise corrected) }}=0.05$, the most significant clusters showing higher mGluR5 binding were found in the hippocampus or parahippocampal gyrus and the orbitofrontal gyrus (Table 2). A comparison of the 6-mo follow-up visit to the baseline condition demonstrated a regional increase in mGluR5 binding similar to that observed at the 2-mo follow-up visit ( $p_{\text {height }}<$ $0.001 ; t>6.5$ ) (Fig. 1B) (Supplemental Table 1).

\section{mGluR5 Availability in Alcohol-Dependent Patients During Abstinence and Healthy Controls}

At the baseline, alcohol-dependent patients demonstrated lower mGluR5 binding in corticolimbic regions (14\%-36\%) than the controls (7). After 2 mo of abstinence, mGluR5 levels in alcoholdependent patients were still significantly lower than those in the 


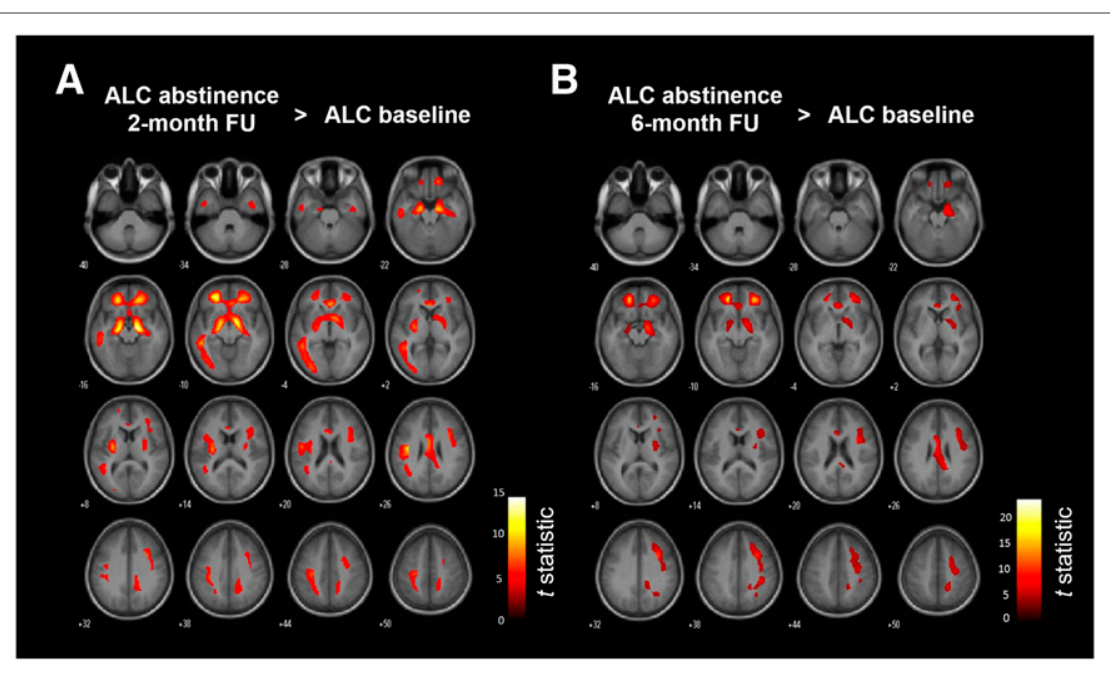

FIGURE 1. SPM12 analysis of increased mGluR5 availability in alcohol-dependent patients (ALC) after 2 mo $(n=10)(\mathrm{A})$ and 6 mo $(n=8)$ (B) of abstinence compared with baseline $(n=$ 16). $F U=$ follow-up.

controls $(16 \%-33 \%)$, particularly in the thalamus $(-33 \% \pm 10 \%$; $P=0.00001$ [Bonferroni corrected]) and the hippocampus $(-28 \% \pm$ $1 \% ; P=0.002$ [Bonferroni corrected]) (Fig. 2). In contrast, at the 6-mo follow-up, mGluR5 availability reached the level seen in the controls in most brain regions $(-6 \% \pm 5 \%$; range of $P$ values, $0.12-0.79)$ (Fig. 2), except for the hippocampus $(-26 \% \pm 21 \%$; $P=0.002)$, thalamus $(-21 \% \pm 20 \% ; P=0.01)$, and nucleus accumbens $(-18 \% \pm 19 \% ; P=0.04)$. At the 2 -mo follow-up, none of the regions had higher $\mathrm{V}_{\mathrm{T}}$ values than the controls. At the 6-mo follow-up, the orbitofrontal cortex was the only region that showed significantly higher $\mathrm{V}_{\mathrm{T}}$ values $(+19 \%$; $P=0.01)$ than the controls. Similar mGluR5 changes were observed when no partial-volume correction data were used (Supplemental Fig. 2), except for the higher $\mathrm{V}_{\mathrm{T}}$ values found in the orbitofrontal cortex at the 6-mo follow-up than in the controls.

\section{Relationships Among mGluR5 \\ Availability, Relapse, and Craving During Follow-Up}

Voxel-based analysis demonstrated that patients who had a relapse during the 6-mo follow-up (relapsers) had higher baseline mGluR5 availability $(+25.1 \%)$ in a cluster located in the anterior putamen and globus pallidus than patients who did not have a relapse (abstainers) (Fig. 3). When the number of drinks per week and the days between the last alcohol consumption and the baseline scan were taken as covariates, the results were unaltered. No significant effects of baseline $V_{T}$ values on the number of drinks per week (Supplemental Table 2) or the number of drinks per drinking day during follow-up were found (all $P \mathrm{~s}>0.3$ ). No changes in hair ethyl glucuronide values during follow-up were found.

There was a positive correlation between the percentage of positive change in mGluR5 binding in striatal regions and the

TABLE 2

Statistical Parametric Mapping of Increased mGluR5 Availability in Alcohol-Dependent Patients After 2 Months of Abstinence

\begin{tabular}{|c|c|c|c|c|c|c|c|}
\hline \multicolumn{2}{|c|}{ Cluster level } & \multicolumn{2}{|c|}{ Voxel level } & \multicolumn{3}{|c|}{ Peak voxel MNI coordinate } & \multirow[b]{2}{*}{ Anatomic area } \\
\hline $\mathrm{K}_{\text {ext }}$ & $P$ & $t$ & $P$ & $x$ & $y$ & $z$ & \\
\hline \multirow[t]{13}{*}{11,805} & $<0.001^{*}$ & 14.7 & $0.002^{*}$ & 16 & -12 & -16 & R parahippocampal gyrus \\
\hline & & 13.8 & $0.004^{*}$ & 14 & -4 & -12 & $\mathrm{R}$ hippocampus \\
\hline & & 13.4 & $0.004^{\star}$ & -24 & 42 & 10 & $\mathrm{~L}$ middle frontal gyrus \\
\hline & & 11.5 & $0.010^{*}$ & -18 & -16 & -16 & L parahippocampal gyrus \\
\hline & & 11.0 & $0.013^{*}$ & -14 & -6 & -12 & L hippocampus \\
\hline & & 10.6 & $0.015^{\star}$ & -38 & -14 & 26 & $\mathrm{~L}$ insula \\
\hline & & 10.2 & $0.020^{*}$ & 20 & 38 & -14 & R superior orbitofrontal gyrus \\
\hline & & 9.2 & $0.035^{\star}$ & -44 & -12 & 24 & $\mathrm{~L}$ insula \\
\hline & & 9.0 & $0.039^{*}$ & 4 & 30 & -4 & $\mathrm{R}$ anterior cingulate cortex \\
\hline & & 8.7 & $0.045^{\star}$ & -28 & -12 & 8 & L putamen \\
\hline & & 7.9 & $1.2 \cdot 10^{-5}$ & -2 & 0 & 26 & $\mathrm{~L}$ middle cingulum \\
\hline & & 7.7 & $1.6 \cdot 10^{-5}$ & -32 & -28 & 40 & L postcentral gyrus \\
\hline & & 7.7 & $1.6 \cdot 10^{-5}$ & 34 & 10 & 28 & $R$ inferior frontal cortex \\
\hline \multirow[t]{3}{*}{2,614} & $0.006^{\star}$ & 9.00 & $0.038^{*}$ & -48 & -40 & -10 & $L$ inferior temporal lobe \\
\hline & & 6.53 & $5.4 \cdot 10^{-5}$ & -50 & -24 & -20 & $L$ inferior temporal lobe \\
\hline & & 6.16 & $8.3 \cdot 10^{-5}$ & -38 & -48 & 24 & $\mathrm{~L}$ angular gyrus \\
\hline
\end{tabular}

${ }^{*}$ Corrected for familywise error.

$\mathrm{MNI}=$ Montreal Neurologic Institute; $\mathrm{K}_{\text {ext }}=$ cluster size extent. 


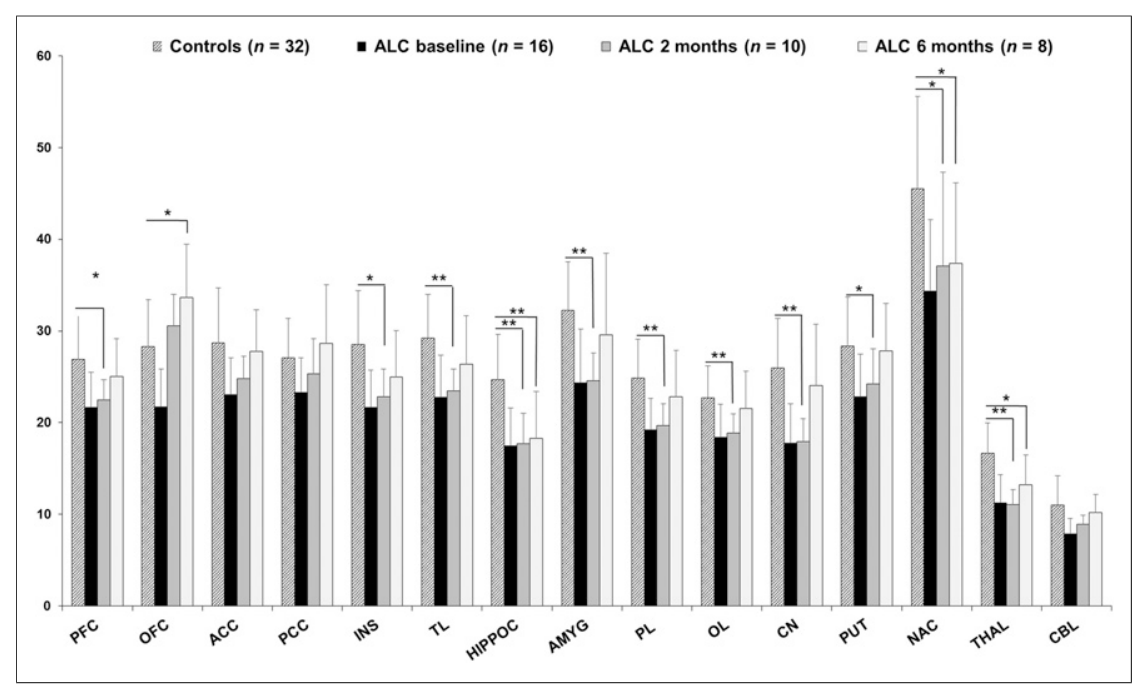

FIGURE 2. Average ${ }^{18} \mathrm{~F}$-FPEB $\mathrm{V}_{\mathrm{T}}$ values in healthy controls and in alcohol-dependent patients (ALC) at baseline and at 2 and 6 mo of follow-up during abstinence. Error bars represent 1 SD. Statistical significances were reported only for ALC at 2 and 6 mo compared with controls; those for ALC baseline vs. controls were reported by Leurquin-Sterk et al. (7). ${ }^{\star} P<0.05$ (2-sided independent $t$ tests). ${ }^{* \star} P<0.05$ (Bonferroni post hoc tests). ACC $=$ anterior cingulate cortex; $A M Y G=$ amygdala; $\mathrm{CBL}=$ cerebellum; $\mathrm{CN}=$ caudate nucleus; $\mathrm{HIPPOC}=$ hippocampus; INS = insula; NAC = nucleus accumbens; OFC = orbitofrontal cortex; $\mathrm{OL}=$ occipital lobe; $\mathrm{PCC}=$ posterior cingulate cortex; $\mathrm{PFC}=$ prefrontal cortex; $\mathrm{PL}=$ parietal lobe; $\mathrm{PUT}=$ putamen; $\mathrm{THAL}=$ thalamus; $\mathrm{TL}=$ temporal lobe. preexisting biologic trait, predisposing them to alcohol dependence, or a state of mGluR5 downregulation. Regardless of whether decreased mGluR5 availability in alcohol-dependent patients is a consequence of alcohol dependence, mGluR5 reduction might influence recovery from the disease. Indeed, excessive glutamatergic neurotransmission associated with alcohol withdrawal and prolonged abstinence is thought to be a central mechanism contributing to craving and relapse (38). Thus, a decrease in postsynaptic mGluR5 could help compensate for this hyperglutamatergic state, thereby improving the odds of patients remaining sober in the long term.

To our knowledge, the present study is the first human study in which mGluR5 was longitudinally assessed in vivo. After just 2 mo and certainly after 6 mo of alcohol detoxification, the recovery of mGluR5 availability in patients achieved levels comparable to those in healthy controls for all brain regions except the hippocampus, nucleus accumbens, and thalamus. In patients with chronic alcohol dependence, persistent senreduction in the craving dimensions "desire and intention to drink" (caudate: $r=0.94, P=0.001$; putamen: $r=0.75, P=0.032$; nucleus accumbens: $r=0.77, P=0.025$ ) and "negative reinforcement" (caudate: $r=0.92, P=0.001$; putamen: $r=0.72, P=0.045$; nucleus accumbens: $r=0.74, P=0.038$ ).

\section{DISCUSSION}

The reduced cerebral mGluR5 availability in alcohol-dependent patients after a severe drinking period (7) represents either a

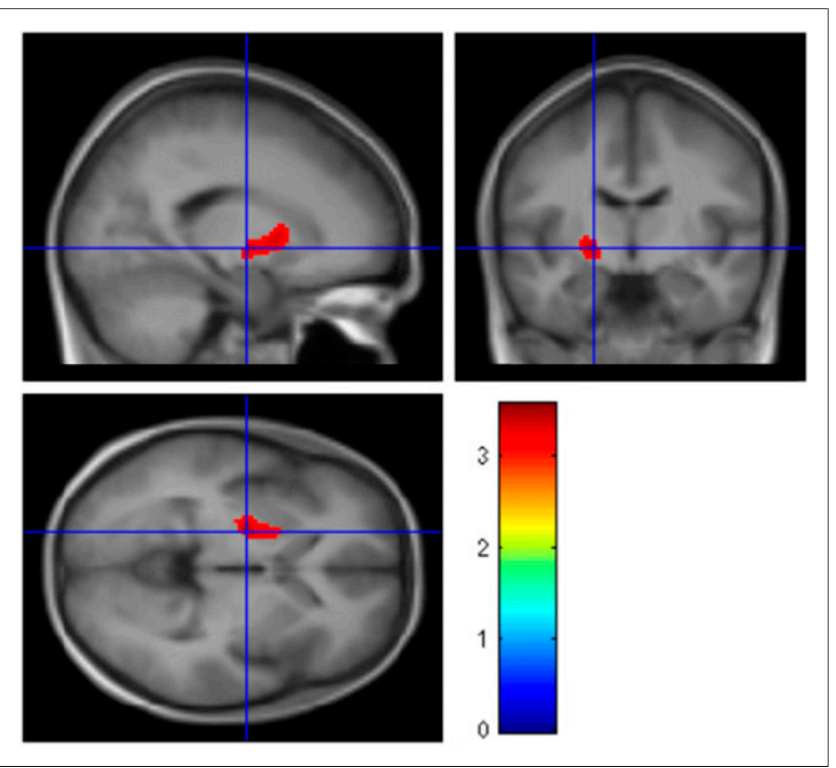

FIGURE 3. SPM12 sagittal, coronal, and sagittal T-maps rendered on a normalized average MRI scan, showing higher mGluR5 availability in patients who had relapse during 6 -mo follow-up period $(n=8)$ than in patients who abstained from alcohol $(n=8)$. sitization of these subcortical limbic-striatal regions-which are involved in reward and memory processes-could compromise the prefrontal regulatory function, in turn affecting recovery from alcohol dependence (39). Overall, these findings support the hypothesis that mGluR5 downregulation represents a lasting but reversible consequence of chronic alcohol consumption, rather than a preexisting trait. However, on the basis of the long-lasting reduction in mGluR5 signaling after 6 mo of abstinence in limbic-striatal regions such as the hippocampus and nucleus accumbens, a specific regional selective mGluR5 decrease might be considered a risk factor for alcohol dependence. In a recent cross-sectional PET study with smokers and ex-smokers, similar reversible effects were suggested (40). These findings are in accordance with preclinical studies suggesting that mGluR5 downregulation represents a common neuroadaptation in the transition from controlled use to dependence for several drugs of abuse $(41,42)$.

A longitudinal small-animal PET experiment with a cocaine self-administration rat model also revealed decreased mGluR5 availability after cocaine exposure, more pronounced in the hippocampus (20). Similarly, ${ }^{18}$ F-FPEB PET revealed that self-administration of alcohol resulted in decreased mGluR5 availability in the hippocampus and amygdala compared with the baseline condition (19). Similarly, extinction from the drug was associated with a full recovery of mGluR 5 availability after $14 \mathrm{~d}$. Interestingly, animals with a lower cocaine-induced reduction in mGluR5 availability, specifically in the hippocampus, showed more severe relapselike behaviors after withdrawal. This observation is consistent with the hypothesis that mGluR5 downregulation represents a beneficial adaptation to chronic drug use, potentially by regulating craving behavior during abstinence. Nevertheless, translation and interpretation of findings derived from animal models of alcohol dependence to humans should be approached with caution.

On the basis of the findings of the present study, some hypotheses can be formulated about the functional and clinical implications of reduced mGluR5 availability in alcohol dependence and its partial 
recovery during abstinence. Within 2 wk of reaching abstinence, lower mGluR5 availability was associated with less craving for alcohol (7). Patients who had a relapse during the 6-mo follow-up period had higher baseline mGluR5 availability than abstainers in a cluster comprising the anterior putamen and internal globus pallidus. These 2 subcortical nuclei are part of the dorsal striatum, which is centrally involved in habit formation and which is the location where lasting drug-induced neuroadaptations driving relapse behaviors are suggested to occur $(16,41,43)$.

Furthermore, better striatal mGluR5 recovery toward normalization was associated with a greater reduction in the desire and intention to drink and negative reinforcement aspects of craving. The direction of this association was unexpected, as lower mGluR5 availability at the start of detoxification was associated with lower levels of craving. A possible explanation is that the normalization of alcohol-induced changes in the glutamatergic state that occurs during abstinence (2124) represents the causal factor for both the recovery of mGluR5 and the reduction in craving.

In a recent longitudinal rat model of alcohol dependence, the prefrontal glutamate concentration decreased during alcohol exposure but normalized after $1 \mathrm{wk}$ of abstinence (19). The full recovery of mGluR5 availability in the hippocampus, nucleus accumbens, and thalamus was not yet achieved at 6-mo after alcohol detoxification. Given the important role of these limbic-striatal regions in regulating addictive behaviors such as emotion regulation, decision making, impulse control, and alcohol craving $(39,44)$, longitudinal studies with longer follow-up periods might be warranted. For instance, lasting impairment of mGluR5-dependent plasticity in these regions might contribute to the relative inabilities of dependent individuals to extinguish alcohol-associated memories and to form adaptive behaviors to prevent alcohol consumption $(45,46)$.

Future studies could also include other behavioral outcomes, such as impaired memory and cognitive control processes, which are inherent to drug dependence $(47,48)$ and which are suggested to depend on the mGluR5 $(45,49)$. Collectively, the present data support the view that modulating mGluR5 signaling by pharmaceutical interventions might be effective in preventing relapse (12-14), as suggested earlier in preclinical studies $(8-10,45,46)$.

Some limitations of the present study merit comment. First, because of the small sample size, the correlations between mGluR5 changes and craving during follow-up should be considered preliminary and await independent replications with larger samples. Another potential confound is the fact that true mGluR5-related relapse risk might depend on the effect of 2 interacting factors. The first is the natural receptor state, in which higher mGluR5 availability might be associated with more novelty seeking (18) and thereby might confer a higher relapse risk. The second is the receptor state after chronic exposure to large amounts of alcohol, which likely downregulates mGluR5; lower mGluR5 availability is associated with less craving and a lower relapse risk. Moreover, although an appealing underlying mechanism for the reversible decrease in mGluR5 availability in patients is the change in glutamatergic activity during abstinence, this hypothesis was not tested here; it could be addressed by combining longitudinal mGluR5 PET with MR spectroscopy of glutamate and its metabolites, in relation to withdrawal severity during early and prolonged abstinence. Indeed, although no straightforward conclusions can be made for an excess or a deficit of available glutamate at the mGluR5 receptor site through the use of MR spectroscopy, combining PET, MR spectroscopy, and receptor blockade might be useful for assessing the effect of modulating mGluR5 signaling on alcohol addiction.

\section{CONCLUSION}

In summary, we found that decreased cerebral mGluR5 availability in alcohol-dependent patients partially recovered during abstinence, with observable changes after just 2 mo. Higher striatal mGluR5 availability was observed in relapsers than in abstainers, and higher mGluR5 recovery was associated with a greater reduction in craving scores during abstinence. Thus, mGluR5 availability could be a marker of disease progression.

\section{DISCLOSURE}

Koen Van Laere is a senior clinical research fellow for the Research Foundation-Flanders (FWO) and has received an FWO research grant for this work (FWO/G.0548.06). Jenny Ceccarini is an FWO postdoctoral fellow. Bart de Laat received a scholarship from the Flemish Agency for Innovation (IWT). No other potential conflict of interest relevant to this article was reported.

\section{ACKNOWLEDGMENTS}

We acknowledge Kwinten Porters, Jef Van Loock, and the radiopharmacy and radiology team at UZ Leuven.

\section{KEY POINTS}

QUESTION: Does the state of decreased metabotropic glutamate receptor 5 (mGluR5) availability in alcohol-dependent patients normalize during abstinence?

PERTINENT FINDINGS: In a case-control study comparing cerebral mGluR5 availability in alcohol-dependent patients during abstinence with that during the recent detoxification (baseline) phase, we found that after 6 mo of detoxification, alcohol-dependent patients showed sustained recovered mGluR5 availability in cortical and subcortical regions compared with the baseline, up to the levels observed in controls, in most areas except for the hippocampus, nucleus accumbens, and thalamus. Also, higher striatal mGluR5 availability was observed in patients who had a relapse (relapsers) than in patients who did not have a relapse (abstainers), and higher mGluR5 recovery was associated with a greater reduction in craving scores during abstinence.

IMPLICATIONS FOR PATIENT CARE: In vivo assessment of cerebral mGluR5 availability could be a potential biomarker in the development of treatments for alcohol dependence.

\section{REFERENCES}

1. Weisner C, Matzger H, Kaskutas LA. How important is treatment? One-year outcomes of treated and untreated alcohol-dependent individuals. Addiction. 2003;98:901-911.

2. Garrison KA, Potenza MN. Neuroimaging and biomarkers in addiction treatment. Curr Psychiatry Rep. 2014;16:513.

3. Leurquin-Sterk G, Postnov A, de Laat B, et al. Kinetic modeling and long-term test-retest reproducibility of the mGluR5 PET tracer ${ }^{18}$ F-FPEB in human brain. Synapse. 2016;70:153-162.

4. Wong DF, Waterhouse R, Kuwabara H, et al. ${ }^{18} \mathrm{~F}-\mathrm{FPEB}$, a PET radiopharmaceutical for quantifying metabotropic glutamate 5 receptors: a first-in-human study of radiochemical safety, biokinetics, and radiation dosimetry. J Nucl Med. 2013;54:388-396.

5. Sullivan JM, Lim K, Labaree D, et al. Kinetic analysis of the metabotropic glutamate subtype 5 tracer $\left[{ }^{18} \mathrm{~F}\right] \mathrm{FPEB}$ in bolus and bolus-plus-constant-infusion studies in humans. J Cereb Blood Flow Metab. 2013;33:532-541.

6. Park E, Sullivan JM, Planeta B, et al. Test-retest reproducibility of the metabotropic glutamate receptor 5 ligand ${ }^{18} \mathrm{~F}-\mathrm{FPEB}$ with bolus plus constant infusion in humans. Eur J Nucl Med Mol Imaging. 2015;42:1530-1541. 
7. Leurquin-Sterk G, Ceccarini J, Crunelle CL, et al. Lower limbic metabotropic glutamate receptor 5 availability in alcohol dependence. J Nucl Med. 2018;59:682-690.

8. Schroeder JP, Overstreet DH, Hodge CW. The mGluR5 antagonist MPEP decreases operant ethanol self-administration during maintenance and after repeated alcohol deprivations in alcohol-preferring (P) rats. Psychopharmacology (Berl). 2005;179: $262-270$.

9. Lee J-Y, Choe ES, Yang CH, et al. The mGluR5 antagonist MPEP suppresses the expression and reinstatement, but not the acquisition, of the ethanol-conditioned place preference in mice. Pharmacol Biochem Behav. 2016;140:33-38.

10. Adams CL, Short JL, Lawrence AJ. Cue-conditioned alcohol seeking in rats following abstinence: involvement of metabotropic glutamate 5 receptors. $\mathrm{Br} \mathrm{J}$ Pharmacol. 2010;159:534-542.

11. Blednov YA, Harris RA. Metabotropic glutamate receptor 5 (mGluR5) regulation of ethanol sedation, dependence and consumption: relationship to acamprosate actions. Int J Neuropsychopharmacol. 2008;11:775-793.

12. Cleva RM, Olive MF, Cleva RM, Olive MF. Positive allosteric modulators of type 5 metabotropic glutamate receptors (mGluR5) and their therapeutic potential for the treatment of CNS disorders. Molecules. 2011;16:2097-2106.

13. Holmes A, Spanagel R, Krystal JH. Glutamatergic targets for new alcohol medications. Psychopharmacology (Berl). 2013;229:539-554.

14. Vadasz C, Saito M. New glutamatergic target for alcohol and substance use disorder medications. Psychopharmacology (Berl). 2014;231:1429-1431.

15. Cleva RM, Hicks MP, Gass JT, et al. mGluR5 positive allosteric modulation enhances extinction learning following cocaine self-administration. Behav Neurosci. 2011;125:10-19.

16. Meyers JL, Salling MC, Almli LM, et al. Frequency of alcohol consumption in humans; the role of metabotropic glutamate receptors and downstream signaling pathways. Transl Psychiatry. 2015;5:e586.

17. Schumann G, Johann M, Frank J, et al. Systematic analysis of glutamatergic neurotransmission genes in alcohol dependence and adolescent risky drinking behavior. Arch Gen Psychiatry. 2008;65:826-838.

18. Leurquin-Sterk G, Van den Stock J, Crunelle CL, et al. Positive association between limbic metabotropic glutamate receptor 5 availability and novelty-seeking temperament in humans: an ${ }^{18}$ F-FPEB PET study. J Nucl Med. 2016;57:1746-1752.

19. de Laat B, Weerasekera A, Leurquin-Sterk G, et al. Effects of alcohol exposure on the glutamatergic system: a combined longitudinal ${ }^{18} \mathrm{~F}$-FPEB and ${ }^{1} \mathrm{H}-\mathrm{MRS}$ study in rats. Addict Biol. 2019;24:696-706.

20. de Laat B, Weerasekera A, Leurquin-Sterk G, et al. Glutamatergic biomarkers for cocaine addiction: a longitudinal study in self-administering rats using MRS and mGluR5 PET. J Nucl Med. 2018;59:952-959.

21. Hermann D, Weber-Fahr W, Sartorius A, et al. Translational magnetic resonance spectroscopy reveals excessive central glutamate levels during alcohol withdrawal in humans and rats. Biol Psychiatry. 2012;71:1015-1021.

22. Bauer J, Pedersen A, Scherbaum N, et al. Craving in alcohol-dependent patients after detoxification is related to glutamatergic dysfunction in the nucleus accumbens and the anterior cingulate cortex. Neuropsychopharmacology. 2013;38:1401-1408.

23. Frischknecht U, Hermann D, Tunc-Skarka N, et al. Negative association between MR-spectroscopic glutamate markers and gray matter volume after alcohol withdrawal in the hippocampus: a translational study in humans and rats. Alcohol Clin Exp Res. 2017;41:323-333.

24. Zahr NM, Rohlfing T, Mayer D, Luong R, Sullivan EV, Pfefferbaum A. Transient CNS responses to repeated binge ethanol treatment. Addict Biol. 2016;21:1199-1216.

25. Umhau JC, Momenan R, Schwandt ML, et al. Effect of acamprosate on magnetic resonance spectroscopy measures of central glutamate in detoxified alcohol-dependent individuals: a randomized controlled experimental medicine study. Arch Gen Psychiatry. 2010;67:1069-1077.

26. Mon A, Durazzo TC, Meyerhoff DJ. Glutamate, GABA, and other cortical metabolite concentrations during early abstinence from alcohol and their associations with neurocognitive changes. Drug Alcohol Depend. 2012;125:27-36.

27. Frye MA, Hinton DJ, Karpyak VM, et al. Anterior cingulate glutamate is reduced by acamprosate treatment in patients with alcohol dependence. J Clin Psychopharmacol. 2016;36:669-674.
28. Love A, James D, Willner P. A comparison of two alcohol craving questionnaires. Addiction. 1998;93:1091-1102.

29. Sobell LC, Sobell MB. Timeline follow-back: a technique for assessing selfreported alcohol consumption. In: Litten RZ, Allen JP, eds. Measuring Alcohol Consumption: Psychosocial and Biochemical Methods. Totowa, NJ: Humana Press; 1992:41-72.

30. Crunelle CL, Yegles M, van Nuijs ALN, et al. Hair ethyl glucuronide levels as a marker for alcohol use and abuse: a review of the current state of the art. Drug Alcohol Depend. 2014;134:1-11.

31. Logan J, Fowler JS, Volkow ND, et al. Graphical analysis of reversible radioligand binding from time activity measurements applied to $\left[\mathrm{N}-{ }^{11} \mathrm{C}\right.$-methyl $]-(-)$-cocaine PET studies in human subjects. J Cereb Blood Flow Metab. 1990;10:740-747.

32. Hammers A, Allom R, Koepp MJ, et al. Three-dimensional maximum probability atlas of the human brain, with particular reference to the temporal lobe. Hum Brain Mapp. 2003;19:224-247.

33. Gazdzinski S, Durazzo TC, Meyerhoff DJ. Temporal dynamics and determinants of whole brain tissue volume changes during recovery from alcohol dependence. Drug Alcohol Depend. 2005;78:263-273.

34. Durazzo TC, Mon A, Gazdzinski S, Yeh P-H, Meyerhoff DJ. Serial longitudinal magnetic resonance imaging data indicate non-linear regional gray matter volume recovery in abstinent alcohol-dependent individuals. Addict Biol. 2015;20:956967.

35. Müller-Gärtner HW, Links JM, Prince JL, et al. Measurement of radiotracer concentration in brain gray matter using positron emission tomography: MRIbased correction for partial volume effects. J Cereb Blood Flow Metab. 1992;12: 571-583.

36. Rousset OG, Ma Y, Evans AC. Correction for partial volume effects in PET: principle and validation. J Nucl Med. 1998;39:904-911.

37. Akkus F, Ametamey SM, Treyer V, et al. Marked global reduction in mGluR5 receptor binding in smokers and ex-smokers determined by $\left[{ }^{11} \mathrm{C}\right] \mathrm{ABP} 688$ positron emission tomography. Proc Natl Acad Sci USA. 2013;110:737-742.

38. Spanagel R. Alcoholism: a systems approach from molecular physiology to addictive behavior. Physiol Rev. 2009;89:649-705.

39. Seo D, Sinha R. Neuroplasticity and predictors of alcohol recovery. Alcohol Res. 2015;37:143-152.

40. Akkus F, Treyer V, Johayem A, et al. Association of long-term nicotine abstinence with normal metabotropic glutamate receptor-5 binding. Biol Psychiatry. 2016;79:474-480.

41. Kalivas PW. The glutamate homeostasis hypothesis of addiction. Nat Rev Neurosci. 2009;10:561-572.

42. Hao Y, Martin-Fardon R, Weiss F. Behavioral and functional evidence of metabotropic glutamate receptor $2 / 3$ and metabotropic glutamate receptor 5 dysregulation in cocaine-escalated rats: factor in the transition to dependence. Biol Psychiatry. 2010;68:240-248.

43. Fuchs RA, Branham RK, See RE. Different neural substrates mediate cocaine seeking after abstinence versus extinction training: a critical role for the dorsolateral caudate-putamen. J Neurosci. 2006;26:3584-3588.

44. Koob GF, Volkow ND. Neurocircuitry of addiction. Neuropsychopharmacology. 2010;35:217-238.

45. Gass JT, Olive MF. Positive allosteric modulation of mGluR5 receptors facilitates extinction of a cocaine contextual memory. Biol Psychiatry. 2009;65:717720.

46. Gass JT, Trantham-Davidson H, Kassab AS, Glen WB, Olive MF, Chandler LJ. Enhancement of extinction learning attenuates ethanol-seeking behavior and alters plasticity in the prefrontal cortex. $J$ Neurosci. 2014;34:7562-7574.

47. Volkow ND, Morales M. The brain on drugs: from reward to addiction. Cell. 2015; 162:712-725.

48. Hyman SE. Addiction: a disease of learning and memory. Am J Psychiatry. 2005;162:1414-1422.

49. Kiritoshi T, Ji G, Neugebauer V. Rescue of impaired mGluR5-driven endocannabinoid signaling restores prefrontal cortical output to inhibit pain in arthritic rats. J Neurosci. 2016;36:837-850. 